\title{
Le corps de ville à Brest : composition, fonctionnement et rôles (1750-1790)
}

The corps de ville in Brest during the Ancien Régime: composition, operation and roles (1750-1790)

\section{Bruno Baron}

\section{OpenEdition}

\section{Journals}

Édition électronique

URL : http://journals.openedition.org/abpo/2573

DOI : 10.4000/abpo.2573

ISBN : 978-2-7535-2782-9

ISSN : 2108-6443

\section{Éditeur}

Presses universitaires de Rennes

\section{Édition imprimée}

Date de publication : 30 mars 2013

Pagination : 129-152

ISBN : 978-2-7535-2780-5

ISSN : 0399-0826

\section{Référence électronique}

Bruno Baron, "Le corps de ville à Brest : composition, fonctionnement et rôles (1750-1790) ", Annales de Bretagne et des Pays de l'Ouest [En ligne], 120-1 | 2013, mis en ligne le 30 mars 2015, consulté le 01 mai 2019. URL : http://journals.openedition.org/abpo/2573; DOI : 10.4000/abpo.2573 


\title{
Le corps de ville à Brest : composition, fonctionnement et rôles (1750-1790)
}

\author{
Bruno BARON \\ CRBC-Université de Bretagne Occidentale (Brest)
}

À Brest, la municipalité est la dernière des institutions, après les commandements de la marine et de l'armée de terre, l'intendance de Bretagne et la sénéchaussée, à prétendre à un pouvoir dans la ville. On ne sait pas grand-chose des institutions municipales brestoises médiévales. Depuis la seconde moitié du Xvi ${ }^{\mathrm{e}}$ siècle au moins, Brest dispose d'une mairie élective ${ }^{1}$; son ralliement en $1589^{2}$ à la cause d'Henri IV et sa résistance à Mercœur et aux Espagnols pendant les guerres de la Ligue lui ont valu, en remerciement, l'obtention en décembre 1593 de lettres patentes qui confèrent à la ville sa première véritable organisation municipale.

Par cet acte de $1593^{3}$, le maire, qui exerçait seul sa fonction, se voit adjoindre deux échevins. Ceux-ci sont élus par « lesdits habitants et bourgeois " pour une période de deux ans. Et " l'un sortira chacun an, au lieu et place duquel en sera choisi un autre par les voix et suffrages desdits habitants ${ }^{4}$. Le roi accorde également aux Brestois le droit de bourgeoisie; pour accéder à cette dignité, une somme de quarante écus ${ }^{5}$ doit être versée dans les caisses du receveur des deniers. La municipalité de Brest s'organise alors autour d'une petite élite locale naissante, comprenant une trentaine de marchands et une dizaine d'hommes de loi. Déjà, ces deux catégories sociales commencent à s'imposer sur le théâtre municipal car ce sont les seules à jouir d'une certaine aisance financière et à pouvoir ainsi payer ce droit d'entrée de reconnaissance bourgeoise.

Mais c'est vraiment à partir de 1681, dans le contexte de grand développement du port, que Brest est reconnue en tant que cité importante du

1. CloÎTRE, Marie-Thérèse (dir.), Histoire de Brest, Brest, CRBC, 2000, p. 49.

2. LE Goff, Hervé, La Ligue en Bretagne. Guerre civile et conflit international (1588-1598), Rennes, PUR, 2010, p. 98.

3. Ce texte a été rédigé lors du Conseil du roi tenu le 31 décembre 1593.

4. Arch. mun. de Brest, AA1-2, lettres patentes du 31 décembre 1593.

5 . Cette somme sert aux réparations et à l'entretien des fortifications. 
royaume. Les lettres patentes de juillet ${ }^{6}$ accordent à la ville l'institution d'un corps municipal. Ce dernier est désormais composé d'un maire, de deux échevins, d'un procureur du roi syndic et de quatre conseillers; un secrétaire-greffier complète cette organisation. Pour régir Brest, il est donc décidé de la création d'un " corps de communauté ". Cette entité sera dirigée par des membres " qui seront choisis pour la première fois entre les plus notables bourgeois de la dite ville ". Afin de permettre à ces administrateurs de disposer de fonds pour " l'entretien du pavé, des quais, murs, portes et ponts de la ville, construction d'une église paroissiale et autres nécessités ", Louis XIV leur accorde un "droit de six deniers pour la pinte de vin vendu en détail ».

De plus, par ces lettres, le bourg de Recouvrance est définitivement rattaché à la ville de Brest " pour jouir par les habitants dudit bourg des mêmes privilèges, droits et prérogatives dont jouissent les habitants de la dite ville ". De même, la cour royale de justice ainsi que les foires et marchés sont définitivement transférés de Saint-Renan ${ }^{7}$ à Brest. Les bases de la construction municipale sont donc posées dès ce moment. À la fin du XVII ${ }^{\mathrm{e}}$ et dans la première moitié du XVIII ${ }^{\mathrm{e}}$ siècle, quelques modifications interviennent toutefois dans l'organisation et le fonctionnement du corps municipal ${ }^{8}$.

En 1750, la municipalité a acquis, pour la somme de 18000 livres, seize charges d'officiers municipaux mises en vente par la monarchie : un maire, deux lieutenants de maire, quatre échevins (deux anciens et deux actifs), quatre assesseurs (deux anciens et deux actifs), deux secrétaires-greffiers, deux contrôleurs des greffes, un procureur du roi ${ }^{9}$. Elle vise à les éteindre et non à leur donner une existence réelle. Après la récupération de cellesci, la municipalité n'a en effet aucunement modifié son fonctionnement et a conservé les charges préexistantes : un maire, deux échevins, quatre conseillers, un conseiller garde-scel, un procureur du roi syndic et son substitut, soit un total de dix officiers, sans compter les membres de l'ancien corps ${ }^{10}$. Les termes de lieutenant de maire, d'assesseurs, de contrôleurs des greffes, spécifiques aux offices créés, n'apparaîtront jamais ensuite

6. Arch. mun. de Brest, AA1-7, lettres patentes de juillet 1681 .

7. Petite ville à une quinzaine de kilomètres au nord-ouest de Brest et qui abritait la sénéchaussée ducale puis royale depuis le Moyen Âge.

8. En 1681, le mandat du maire est porté à trois ans. En 1701, les fonctions de substitut du procureur-syndic et de conseiller garde-scel sont créées. En 1747, François Jourdain, membre du corps de ville, achète, au titre de la communauté, la charge de maire qui devient dès lors de nouveau élective et en 1748, c'est Antoine Raby qui se porte acquéreur, toujours au titre de la communauté, de tous les autres offices municipaux mis en vente par Louis XV. Pour ces deux Brestois, cette action se traduit par l'impossibilité de prétendre à occuper le poste de maire pour Jourdain et un des sièges d'édiles cooptés pour Raby.

9. Arch. mun. de Brest, BB33, reçu du 10 avril 1750.

10. Depuis 1701, les anciens édiles sont membres de droit du corps de ville. Dans la seconde moitié du XVIII ${ }^{\mathrm{e}}$ siècle, 27 individus assistent aux débats du corps de ville en tant que membres de l'ancien corps et selon les périodes, ce nombre de présents oscille 
dans l'organigramme municipal. L'achat des offices visait seulement à les faire disparaître définitivement.

Dans la seconde partie du XVIII ${ }^{\mathrm{e}}$ siècle, l'organisation municipale met en évidence trois ensembles au poids et aux rôles bien différents : le corps en exercice, au sein duquel le maire et secondairement le procureur-syndic occupent le premier plan, et l'ancien corps où se retrouvent potentiellement tous ceux qui ont occupé des charges municipales ainsi qu'un personnel technique réduit au miseur ${ }^{11}$ et au greffier.

\section{Le maire : au sommet de la hiérarchie municipale}

La fonction de maire donne à son titulaire des prérogatives, des droits et des devoirs. Mais l'ensemble de ces pouvoirs ne représente pas une réelle autorité comparée à celle détenue par les agents du pouvoir royal présents en ville. Cela tient plus, en effet, du domaine de l'honorifique que d'une réelle emprise et domination sur la population.

Son statut politique confère au maire quelques prérogatives au sein de la société brestoise. Il a le privilège d'allumer les feux de joie dans le cadre des festivités organisées pour un événement profane ou sacré. Lors des processions, il occupe la tête du cortège à la gauche du sénéchal. Au cours des services religieux, il est au premier rang et reçoit la communion en deuxième position après le sénéchal. Mais cet ordre des choses est bouleversé si le commandant de la place et/ou de la marine assistent à une messe ou à une cérémonie officielle. Dans ces cas, le maire se trouve relégué en une position nettement moins flatteuse en regard de son rang de premier magistrat de la cité. L'édile s'efface devant le représentant du roi ou de la haute noblesse, confirmant une hiérarchisation bien établie des pouvoirs.

Lors des diverses cérémonies, il arbore une tenue d'apparat. Comme les autres officiers municipaux, il porte une toque de velours et revêt une robe $^{12}$, signe distinctif de la magistrature. Cet habit sert principalement lors des Te Deum et des réceptions de personnalités de haut rang. Par contre, aucun portrait officiel n'est établi aux frais de l'administration, atténuant ainsi la visibilité de l'institution municipale.

Le jour de son investiture, il devient colonel de la milice bourgeoise. Mais ne pouvant pas toujours s'occuper réellement de cette tâche, il délègue ce pouvoir à une autre personne qui prend le titre de lieutenant-colonel ou major. Depuis 1754, le maire possède aussi le titre de lieutenant général de police, en charge du tribunal du même nom. En présence du sénéchal, il préside hebdomadairement les séances où l'on aborde la police des mar-

entre trois et onze personnes, ce sont les anciens maires qui sont les plus assidus mais la régularité de leur participation ne répond à aucune règle définie.

11. Dans les municipalités bretonnes, le terme de "miseur " est presque toujours préféré à celui de trésorier.

12. Arch. mun. de Brest, 2S30, règlement du 6 décembre 1618. 
chés, le contrôle des métiers ou les questions sanitaires ${ }^{13}$. Non sans mal, car les sénéchaux n'ont guère d'estime pour ces hommes qui ont acquis cet office suite à un procès. En 1752, le corps de ville demande l'autorisation à l'intendant de s'adresser au conseil du roi pour " faire la levée à son profit des charges de lieutenant général et de procureur du Roi de police de Brest [...] de la manière qu'elles sont exercées dans les autres villes de la province ${ }^{14}$ ". Les lettres patentes du 11 mars 1754 réunissent l'office de lieutenant général de police à la communauté et pour les obtenir, la ville a payé 1650 livres aux parties casuelles. Les officiers municipaux ont obtenu gain de cause car ils ont rappelé qu' " un règlement du 10 août 1683 " précisait qu'à Brest " la police serait faite par le sénéchal avec les maire et échevins ${ }^{15}$ ". Ce rattachement arrive assez tard car des cités, comme Rennes en 1706, Morlaix en 1710 ou Nantes en $1720^{16}$, n'ont pas attendu pour acquérir cet office, même si cet achat ne s'est pas fait sans heurt comme à Morlaix ou à Vitré où les luttes entre corps de ville et juges ont duré plusieurs décennies ${ }^{17}$.

Pour les séances du conseil de la communauté, le maire fixe l'ordre du jour, dirige et oriente les débats. En tant que premier magistrat, il reçoit le serment de tous les officiers municipaux lors de leur installation. Pour seule compensation financière, il perçoit trois cents livres de gages par an, auxquels s'ajoutent cinquante livres pour les frais de bureau. Les autres membres de la municipalité n'ont pas d'émoluments car le maire supporte souvent seul le fardeau quotidien de l'administration. Il est seulement aidé par le secrétaire-greffier et des commis au statut de clerc-praticien pour l'expédition des affaires courantes.

Depuis l'édit de 1681 qui stipule que " le maire de notre dite ville sera appelé à la tenue des États de la Province pour y avoir séance et voix délibérative ${ }^{18}$ ", il est le représentant désigné de la communauté de ville aux États de Bretagne. Cette présence obligatoire n'est pas toujours chose facile car cela l'amène à quitter ses affaires pour quelques mois. Les débats de l'assemblée provinciale durent plusieurs semaines, voire jusqu'à six mois ${ }^{19}$. Le député de la communauté doit avoir le loisir de pouvoir s'absenter. Cet éloignement forcé n'est pas toujours bien vécu,

13. SAUPIN, Guy, « Le pouvoir municipal en France à l'époque moderne. Bilan historiographique des vingt dernières années ", dans Le Pouvoir municipal de la fin du Moyen Âge à 1789, Philippe Hamon et Catherine LaURENT (éd.), Rennes, PUR, 2012, p. 15-54.

14. Le rédacteur fait ici sans doute référence à des villes comme Nantes, Rennes ou Morlaix qui possèdent ces charges depuis le premier quart du XVIII ${ }^{\mathrm{e}}$ siècle. Arch. mun. de Brest, BB33, courrier du 27 juillet 1752 .

15. Arch. mun. de Brest, BB33, arrêt du Conseil du roi du 19 février 1754.

16. Deborde-Lissillour, Séverine, Les Sénéchaussées royales de Bretagne. La monarchie d'Ancien Régime et ses juridictions ordinaires (1532-1790), Rennes, PUR, 2006, p. 171.

17. SAUPIN, Guy (dir.), Histoire sociale du politique. Les villes de l'Ouest atlantique français à l'époque moderne (XVI'-XVIII siècle), Rennes, PUR, 2010, p. 40-42.

18. Arch. mun. de Brest, AA1-7, lettres patentes de juillet 1681 .

19. Pour la période de 1750 à 1788, la session la plus courte, celle de 1754 ne dure que 50 jours. Toutes les autres dépassent les deux mois, 10 dépassant les trois mois et les 
car pendant ce temps, les affaires de l'intéressé doivent continuer à fonctionner. Si la ville prend en charge les frais journaliers ${ }^{20}$ de son député, celui-ci ne perçoit aucune compensation financière pour sa députation. Il est donc impératif pour la personne qui occupe cette fonction d'avoir une certaine aisance financière.

De plus, le premier édile avance souvent l'argent nécessaire aux dépenses de la communauté; il ne rentre dans ses fonds qu'une fois que le miseur a reçu l'ordre de procéder aux remboursements des sommes, soit dans un délai compris entre un et trois ans après que le maire ait engagé son argent personnel ${ }^{21}$. Entre 1751 et 1789 , la somme avancée, sur une année, varie de 515 livres à 3890 livres. Cette possibilité n'étant pas à la portée de toutes les bourses, ces dépenses presque inhérentes à la fonction de maire ont peut-être freiné plus d'un notable brestois dans ses aspirations politiques.

Les considérations pécuniaires ne sont pas le seul butoir; la lourdeur de la tâche en représente un autre. Le premier magistrat doit disposer d'une grande disponibilité et doit pouvoir se libérer de ses occupations professionnelles. Ce poste implique une participation quotidienne aux affaires de la cité.

Un des devoirs qui incombent également au maire consiste à gérer le casernement des troupes et la distribution des billets de logement. Dans la seconde moitié du XVIII ${ }^{\mathrm{e}}$ siècle, le processus d'attribution des divers logis suit quasiment toujours le même chemin. L'information initiale émane du gouverneur militaire de la province et/ou de l'intendant, selon la présence en Bretagne de ces personnages, qui transmettent au commandant de la ville, au maire et/ou au correspondant de la commission intermédiaire des États, les besoins en hébergement pour le ou les régiments qui vont arriver. Dans tous les cas, le commandant de la place se décharge de cette mission sur le premier édile qui, avec l'aide des services administratifs de la municipalité, établit les billets de logement, qui sont ensuite remis aux officiers des différents bataillons. Cette tâche, très ingrate, vaut d'ailleurs au maire plusieurs soucis, contribuant à le rendre parfois impopulaire car les habitants ne sont jamais d'accord avec la répartition : les reproches fusent, les plaintes s'accumulent, la jalousie éclate au grand jour. Car si le maire est dispensé de cet impôt, quelques notables voudraient bien aussi profiter de ce privilège. À Brest, seul le maire est exempté de cette obliga-

\footnotetext{
deux plus longues, celles de 1766 et 1764, dans le contexte très particulier de l'affaire de Bretagne, durent respectivement 146 et 183 jours (soit 6 mois).

20. À son retour, le maire présente au corps municipal les frais engendrés par sa présence aux États. Après acceptation du conseil de la communauté, demande est faite au duc de Penthièvre que le miseur puisse rembourser l'intéressé. Cela apparaît dans les registres des comptes du miseur (Arch. mun. de Brest, registres CC128 et 129) mais le remboursement n'est souvent effectif qu'après un, voire deux ans.

21. Arch. mun. de Brest, CC128 et 129, registres des comptes du miseur (1733-1788).
} 
tion, tandis qu'à Nantes ${ }^{22}$ c'est l'ensemble du corps de ville qui bénéficie de ce privilège. Mais, dans une ville comme Brest, la fréquence et le nombre des casernements expliquent à la fois que l'on octroie peu d'exemption et qu'on en réclame beaucoup.

Depuis janvier 1689, le mandat du maire est de trois ans et avant de procéder à son remplacement lors d'une élection, deux étapes se déroulent. Pour atteindre la fonction de maire, les postulants doivent passer par un processus électoral. Choisi parmi trois candidats, le maire est élu par une assemblée générale ne regroupant qu'une infime partie de la population. Cette réunion a souvent donné lieu à des oppositions diverses mais ces altercations n'ont jamais pu altérer le déroulement du processus électoral, toutes les sessions ayant toujours abouti à un résultat.

Tout d'abord, le corps de ville désigne trois candidats puis l'assemblée générale élit le maire. Le choix des prétendants se fait en respectant le principe de l'alternance entre les deux côtés de la ville. Théoriquement, sur une période de neuf ans, les maires issus de la paroisse Saint-Louis exercent durant six années et celui venant du côté de Recouvrance est en place trois ans. Durant toute la seconde moitié du XVIII ${ }^{\mathrm{e}}$ siècle, ce principe de l'alternance a toujours été scrupuleusement respecté.

Les personnages pressentis pour concourir sont choisis parmi les échevins ou conseillers du corps en exercice ou de l'ancien corps; il ne peut être fait appel à aucune personne extérieure mais rien n'interdit qu'un maire puisse se représenter. Cette possibilité de se voir offrir un nouveau mandat n'existe pas partout dans le royaume; ainsi, à Chartres ${ }^{23}$, à partir de 1765 et à la suite de la réforme L'Averdy, la charge de maire n'est pas renouvelable; à l'inverse à Angers, le maire est rééligible ${ }^{24}$.

La nomination des candidats à l'élection donne lieu, régulièrement, à des débats houleux lors de la séance municipale dédiée à cette fin car les prétendants ne sont pas très nombreux et certains ne veulent pas prendre le risque d'être promu au poste de maire. Quand la liste des trois postulants est arrêtée, elle est transmise au gouverneur de Bretagne, qui entérine ou pas la décision du conseil de la communauté. L'obtention de l'accord du gouverneur est impérative pour l'organisation des élections. La convocation de l'assemblée générale pour l'élection du maire est annoncée aux prônes des grandes messes.

22. SAUPIN, Guy, Nantes au XVII siècle, vie politique et société urbaine, Rennes, PUR, 1996, p. $273-276$.

23. GARnOt, Benoît, Un déclin : Chartres au XVIII siècle, Paris, CTHS, 1991, p. 127.

24. Maillard, Jacques, Le Pouvoir municipal à Angers de 1657 à 1789, 2 tomes, Angers, PU Angers, 1984, p. 27-34. 
Pour l'élection, deux catégories d'individus ont le droit de vote : deux députés ${ }^{25}$ de chaque corps des arts et métiers ${ }^{26}$ qui doivent savoir lire et écrire, et des ayants-droit que sont les officiers municipaux en poste, les membres de l'ancien corps, les notaires royaux, les officiers de la milice bourgeoise, les officiers grenadiers, les avocats, le prieur, les recteurs de Saint-Louis et de Saint-Sauveur, les marguilliers des deux paroisses (anciens et nouveaux), le subdélégué de l'intendant, les juges royaux, les procureurs, les directeurs des hôpitaux (anciens et nouveaux), les huissiers, les greffiers royaux, ce qui offre aux gens de justice et aux notables revêtus de responsabilités civiques une dimension importante dans la cité. Le jour de l'élection, les délégués des corporations sont les seuls à devoir se présenter au procureur-syndic du roi afin de faire enregistrer leur participation. Ils sont munis de la délibération du corps de leur communauté professionnelle qui stipule le nom du candidat sur lequel ils portent leur suffrage. Toutefois, tout comme dans la vie politique de la ville ${ }^{27}$, les représentants des corps de métiers ne constituent pas un poids important dans cette élection, à la différence de ce qui existe dans les cités du nord du royaume ${ }^{28}$. Cet amalgame des forces d'encadrement et des délégués des métiers représente " un compromis entre le désir de réserver les droits civiques à la véritable bourgeoisie urbaine et la volonté d'intégrer l'élite populaire des maîtres de métiers ${ }^{29} "$.

Le nombre potentiel d'électeurs sur la liste dressée par le procureursyndic le jour du scrutin, varie de 129 à 147 durant la seconde moitié du XVIII ${ }^{\mathrm{e}}$ siècle, ce qui est minime par rapport à l'ensemble de la population, ces électeurs ne représentent que $1 \%$ à $1,2 \%$ des chefs de feu. Cette assemblée générale est considérée comme fermée car elle est limitée à une certaine catégorie d'individus. À l'inverse, celles de Nantes ou de Dijon sont dites ouvertes car elles accueillent tous les chefs de feu. À Nantes, l'assemblée générale attire entre 450 et 600 votants. Par contre, dans les cas nantais

25. Le même nombre de représentants des corps de métiers se retrouve à Angers (MAILlARD, Jacques, Le Pouvoir municipal à Angers..., op. cit., p. 87-90).

26. Les corporations qui peuvent être représentées sont : les serruriers, les cordonniers, les boulonniers, les tailleurs, les armuriers-arquebusiers, les fourbisseurs, les barbiersperruquiers, les marchands-épiciers, les graveurs sur métaux, les orfèvres, les chirurgiens, les marchands de draps, les chaudronniers, les bouchers, les maréchaux-ferrants, les taillandiers, les cloutiers, les couteliers, les couvreurs, les maçons, les boulangers, les charpentiers, les menuisiers, les tourneurs, les molletiers-cartiers, les tapissiers, les traiteurs et pâtissiers, les chandelliers, les plombiers et potiers d'étain, les marchands ciriers, les soudeurs, les horlogers, les vitriers, les marchands libraires, les marchands de vin, les apothicaires, les imprimeurs, les négociants (Arch. mun. de Brest, BB29, P.V. d'élections).

27. SAUPIN, Guy (dir.), Histoire sociale du politique..., op. cit., p. 64-65.

28. LEYSSENS, Agathe, "Le pouvoir dans la ville. De l'utilisation des corps de métiers par le Magistrat de Dunkerque ", dans Le Pouvoir municipal..., op. cit., p. 145-162.

29. SAUPIN, Guy, «Élite sociale et pouvoir municipal dans les villes littorales de Bretagne aux Temps modernes ", dans Pouvoirs et littoraux du XVe au XXe siècle. Actes du colloque international de Lorient (24, 25, 26 septembre 1998), Gérard LE BouËDEC et François CHAPPÉE (éd.), Rennes, PUR, 2000, p. 85-97. 
et dijonnais, les participants ne votent pas pour élire un maire mais pour établir une liste de candidats à transmettre au roi ${ }^{30}$.

La participation fluctue selon les époques. Pour des raisons pratiques (occupations professionnelles, raisons de santé) ou conjoncturelles (problème d'alphabétisation, indifférence), certains ne se déplacent pas. En moyenne, la moitié des personnes habilitées à voter ne fréquentent pas ces assemblées générales. Le taux de participation doit également être relativisé par la notion de cumul car un même individu peut entrer dans différentes catégories ${ }^{31}$. La fonction de maire concentrant l'essence même du pouvoir municipal, la réunion électorale attire tous ceux qui s'intéressent ardemment au rayonnement, au rôle et à la place de l'institution dans la société brestoise mais motive moins les groupes dont la participation au système d'honorabilité locale est plus aléatoire.

Aucun élément ne permet de comprendre l'attitude des électeurs, aucun critère de comparaison (nature des candidats, jour de l'élection, conjoncture du moment...) ne donne des outils pour trouver une ou des explications tangibles à ce comportement électoral. De plus, ces scrutins ne reflètent en rien la réalité démographique brestoise. La majorité de la population est écartée du vote, et $a$ fortiori des décisions qui en découlent. Cela se rapproche d'une " mascarade électorale ${ }^{32}$ " dont l'historien peine à démêler les tenants et les aboutissants.

Le jour de l'élection, le président de séance ${ }^{33}$ procède à l'appel des votants. Une fois le nom des candidats inscrit sur une feuille, chaque électeur passe au bureau pour apposer un trait devant le patronyme de son choix. Ce procédé simpliste a été appliqué durant tout l'Ancien Régime : à Nantes comme à Brest, ce principe s'appelle "faire la pique ${ }^{34}$ ". En 1777, Charles-Louis Gillart ${ }^{35}$ a pourtant essayé de changer ce mode de fonctionnement, en proposant le remplacement de la feuille de pointage par des bulletins ${ }^{36}$ mais cette demande a été rejetée. Dix ans plus tard, un autre procureur-syndic, François-Marie Guesnet, réitère cette requête : "pour établir une liberté entière sur ce point essentiel, nous proposons un scrutin secret; nous laissons à la sagesse de l'assemblée à en convenir. Nous aimons à croire qu'elle nous saura gré de notre proposition ${ }^{37}$ ". Une fois de

30. SAUPIN, Guy, "La réforme des institutions municipales en France au XVIII ${ }^{\mathrm{e}}$ siècle : réflexion à partir de l'exemple nantais ", Revue d'Histoire Moderne et Contemporaine, tome 46-4, 1999, p. 629-657. GRAS, Pierre (dir.), Histoire de Dijon, Toulouse, Privat, 1987, p. 166.

31. Il n'est pas rare de voir un même individu être à la fois délégué d'un corps de métiers, marguillier d'une paroisse et officier de la milice bourgeoise, mais cela ne lui confère cependant la possibilité que de voter une seule fois.

32. Coste, Laurent, Messieurs de Bordeaux. Pouvoirs et hommes de pouvoirs à l'hôtel de ville (1548-1789), Bordeaux, CAHMC-FSHO, 2006, p. 105.

33. La séance est présidée par le commandant de la ville ou en son absence par le sénéchal.

34. SAUPIN, Guy, Nantes au XVIז siècle..., op. cit., p. 85.

35. Il est procureur-syndic de la communauté.

36. Arch. mun. de Brest, BB23, assemblée du 11 juin 1777.

37. Arch. mun. de Brest, BB25, assemblée du 4 août 1787. 
Résultats de l'élection des maires (1750-1789)

\begin{tabular}{|c|c|c|c|}
\hline Années & Candidats & Nbre de voix & $\begin{array}{c}\text { Pourcentage } \\
\text { des voix }\end{array}$ \\
\hline \multirow{3}{*}{1750} & Champerault & 16 & 21,0 \\
\hline & Jourdain & 9 & 11,9 \\
\hline & Labbé & 51 & 67,1 \\
\hline \multirow{3}{*}{1753} & Debon & 56 & 58,3 \\
\hline & Le Milbéo & 24 & 25,0 \\
\hline & Martret-Depréville & 16 & 16,7 \\
\hline \multirow{3}{*}{1756} & Debon & 20 & 31,7 \\
\hline & Martret-Depréville & 38 & 60,3 \\
\hline & Raby Antoine & 5 & 8,0 \\
\hline \multirow{3}{*}{1759} & Floch-Maisonneuve & 5 & 7,4 \\
\hline & Jourdain & 23 & 33,8 \\
\hline & Lunven-Kerbizodec & 40 & 58,8 \\
\hline \multirow{3}{*}{1762} & Demontreux & 3 & 4,7 \\
\hline & Féburier & 47 & 72,3 \\
\hline & Raby Antoine & 15 & 23,0 \\
\hline \multirow{3}{*}{1766} & Demontreux & 7 & 9,3 \\
\hline & Malassis & 30 & 39,4 \\
\hline & Raby Antoine & 39 & 51,3 \\
\hline \multirow{2}{*}{1769} & Lunven-Kerbizodec & 70 & 88,6 \\
\hline & Floch-Maisonneuve & 9 & 11,4 \\
\hline \multirow{2}{*}{1771} & Bérubé-Costantin & 21 & 28,0 \\
\hline & Le Normand & 54 & 72,0 \\
\hline \multirow{3}{*}{1777} & Kerbrezan-Cabon & 19 & 25,6 \\
\hline & Le Guen François & 12 & 16,3 \\
\hline & Le Guen Louis & 43 & 58,1 \\
\hline \multirow{2}{*}{1783} & Guesnet & 32 & 45,5 \\
\hline & Raby François & 34 & 54,5 \\
\hline \multirow{3}{*}{1787} & Floch-Maisonneuve & 27 & 33,3 \\
\hline & Le Guen François & 47 & $\mathbf{5 8 , 0}$ \\
\hline & Michel & 7 & 8,7 \\
\hline \multirow{3}{*}{1789} & Branda & 35 & 53,8 \\
\hline & Guesnet & 29 & 44,6 \\
\hline & Le Gléau & 1 & 1,6 \\
\hline
\end{tabular}


plus, les membres présents à l'assemblée générale ne veulent pas utiliser un autre procédé ${ }^{38}$. Ce mode a toujours existé et les votants n'envisagent pas d'en changer. Cette assemblée ne donne ordinairement lieu à aucun débat $^{39}$. Le maire est élu sur sa personnalité, sa fonction, sa fortune et ses valeurs. À l'issue du scrutin, le candidat ayant obtenu le plus de suffrage est proclamé maire de la ville.

L'élection d'un maire est le seul moment où certaines composantes de la société peuvent s'exprimer. Mais dans le cas brestois, l'expression est limitée à sa plus simple représentation. Une infime partie de la population est appelée à voter. Les débats de fond sont absents - ou du moins n'apparaissent pas dans les procès-verbaux -, les candidats sont élus uniquement sur la perception que les votants ont d'eux, sur l'image qu'ils dégagent et sans doute sur leur réputation générale dans la ville.

Que l'on soit en présence de deux ou trois candidats, le maire est à chaque fois élu en obtenant la majorité absolue. Les chiffres varient toutefois fortement de 51,3\% des suffrages exprimés en 1766 à 88,6 \% en 1769 .

Certains postulants n'obtiennent que très peu de voix. Ils montrent sans doute peu d'intérêt pour occuper le poste de maire et ont peut-être fait savoir à leur entourage qu'ils n'étaient pas disposés à consacrer du temps à cette fonction. Les résultats laissent apparaître à chaque fois un grand favori qui l'emporte sans aucune difficulté, sauf en juin 1783 où les deux prétendants ne sont départagés que par deux voix d'écart. Mais "l'état d'esprit de l'époque réfute l'idée même de candidature en lui préférant la notion d'émergence naturelle du futur magistrat ". " Est-ce à dire que les votes obtenus à l'unanimité ou sans incident n'ont été précédés d'aucun conciliabule, d'aucune concertation ${ }^{40}$ ? " Il n'y a point de traces écrites de tels agissements; pourtant rien n'interdit de penser que des démarches pré-électorales ont pu être menées.

\section{Une organisation municipale hiérarchisée}

Le maire préside aux affaires de la cité, il s'appuie pour cela sur une équipe d'officiers municipaux formant le corps en exercice et dans lequel la hiérarchie traduit l'importance des fonctions. La progression interne au sein du corps permet d'appréhender les carrières des édiles. Le corps de ville est complété par les anciens maires, échevins, procureurs et conseillers qui restent membres de droit de la municipalité après leur sortie

\footnotetext{
38. Les archives n'offrent aucune valeur chiffrée pour connaître les résultats exprimés pour ces deux demandes.

39. Il n'y a pas de débat sur le fond mais sur la forme avec lutte pour la présidence entre le sénéchal et le maire en l'absence du commandant de la ville, avec des reproches sur l'organisation, etc.

40. . Coste, Laurent, «Être candidat aux élections municipales dans la France d'Ancien Régime ", dans Le Pouvoir municipal..., op. cit., p. 209-219.
} 
du corps en exercice. À cet ensemble s'ajoutent un responsable de la caisse de la communauté (le miseur) et un secrétaire administratif (le greffier).

À côté du maire, le corps en exercice se compose de deux échevins, quatre conseillers ordinaires, un conseiller garde-scel, un procureur-syndic du roi et son substitut. Tous les membres intègrent le corps selon le principe de la cooptation. À la différence du maire, ils ne passent par aucune forme de scrutin, ce qui laisse une grande liberté aux notables pour choisir leurs nouveaux collègues. Ces nominations sont entérinées par le duc de Penthièvre. Ce processus diffère par exemple de celui rencontré à Nantes où tous les postes sont soumis au vote car que ce soit pour les places d'échevin ou de procureur, une liste est transmise au roi pour désignation finale ${ }^{41}$.

Ce procédé brestois confère aux officiers municipaux, dès leur première cooptation, un pouvoir de siéger en tant qu'administrateur jusqu'à leur mort ou leur démission. Si les critères de désignation manquent de précision, tous ces hommes bénéficient soit d'une aisance financière (négociant-marchand), soit d'une fonction de prestige (homme de loi), soit d'une profession en vue (médecin, apothicaire, imprimeur etc.) D'ailleurs en septembre 1787, le maire François Le Guen précise avant de procéder à la nomination que "le choix ne doit tomber que sur des personnes d'une profession honnête, d'une probité intacte et généralement connue d'une capacité qui les rende propre à remplir avec un zèle éclairé les diverses fonctions et charges publiques qu'entraîne la municipalité ${ }^{42}$ ".

Quand l'ensemble du corps en exercice est au complet, la répartition des rôles a lieu. L'attribution des fonctions et des rangs se fait sous la forme d'un vote interne, le rituel observé est toujours le même : chaque notable coopté inscrit un nom sur un billet et le dépose dans un chapeau tenu par le maire. La personne ayant obtenu le plus de suffrages est installée dans la fonction. Une fois élus, les officiers municipaux prêtent un serment devant le maire dont les actes rapportent la formule : "Promis et juré de se bien et fidèlement comporter dans leurs fonctions ${ }^{43}$."

Les premiers à suivre hiérarchiquement le maire sont les échevins. Étant classés premier et second, ils sont les remplaçants directs du maire. En cas d'absence de ce dernier, ils sont appelés à diriger les séances du conseil. L'ordre est toujours respecté, le premier échevin ayant priorité sur le second. Cependant, quand le maire est absent, l'activité municipale s'en ressent avec une baisse de la périodicité des séances et du nombre de courriers expédiés.

Durant la seconde moitié du XVIII ${ }^{\mathrm{e}}$ siècle, quand le premier magistrat ne peut assurer la présidence ${ }^{44}$, les réunions s'espacent et diminuent

41. SAUPIN Guy, Nantes au XVII siècle..., op. cit., p. 75.

42. Arch. mun. de Brest, BB25, séance du 13 septembre 1787.

43. Arch. mun. de Brest, BB29-105, séance du 28 juin 1777.

44. Les absences sont généralement justifiées : présence aux États, affaires professionnelles ou incommodités personnelles. 
en moyenne de moitié ${ }^{45}$. Par exemple, en 1752, pendant que Guillaume Labbé assiste aux États de Bretagne de fin septembre à fin décembre, il n'y a qu'une réunion mensuelle contre trois auparavant, et en 1770 lors de l'absence de Jean-Pierre Lunven-Kerbizodec, le rythme des réunions passe de quatre à une. Par contre, durant les États qui se tiennent de fin décembre 1766 à fin mai 1767 et auxquels participe Antoine Raby, le premier échevin Georges Demontreux maintient la périodicité des séances. De même, pendant ces périodes d'absence, et malgré l'importance des sujets traités, la correspondance perd aussi de l'ampleur : pour la période 17711783 et lors de la présence du maire aux États, l'envoi des courriers baisse de trois quarts ${ }^{46}$. Les échevins, même s'ils ont autorité pour présider les séances du corps de ville, n'ont pas la même motivation que le maire pour diriger les affaires de la cité. Peut-être craignent-ils aussi de prendre des décisions qui lui déplairont? En tout cas, ils semblent presque se limiter à gérer les affaires courantes et toutes les décisions importantes ne sont prises qu'au retour du maire. Tout se passe comme si la vie politique provinciale se substituait momentanément à la gestion municipale habituelle.

L'autre personnage fondamental de la vie municipale est le procureur du roi syndic. Cette fonction a été établie par les lettres patentes de 1681 et la ville de Brest bénéficie de cette charge quelques années avant Bayeux mais bien après Nantes ${ }^{47}$. Le procureur-syndic est chargé de faire appliquer les lois et règlements en vigueur et de défendre les intérêts de la ville, il a pour mission d'être le représentant du souverain au sein de l'institution; aucune décision n'est arrêtée " sans avoir entendu ses conclusions qui précédaient immédiatement le vote ${ }^{48} »$. Durant son mandat, il est appelé à vérifier si toutes les délibérations sont prises conformément aux règles, il est aidé pour cela par le secrétaire-greffier qui est toujours de formation juridique et il est ainsi le chef d'une administration embryonnaire. Mais le procureur-syndic n'est qu'un intermédiaire car, le plus souvent, ce sont les services du gouverneur ou de l'intendant qui étudient les différents cas de figure. Pourtant, plusieurs détails démontrent que cet homme a la possibilité de faire appliquer les mesures. Tant que cela ne sort pas du cadre local, le procureur-syndic a toutes les libertés pour exiger que les textes ne soient pas bafoués. Il a une très grande connaissance des dossiers et ne craint pas de s'opposer aux autres édiles. L'affrontement est parfois âpre mais tous les hommes qui ont exercé ce rôle l'ont toujours fait avec beaucoup de discernement. En cas d'absence, le procureur-syndic est remplacé par un substitut.

45. Calcul établi à partir des données relevées dans les registres de délibérations de la série BB des Archives municipales de Brest.

46. Calcul établi à partir des données relevées dans les registres de délibérations et de correspondance de la série BB des Archives municipales de Brest.

47. EL Kordi, Mohamed, Bayeux aux XVII et XVIII siècles, Paris, Mouton, 1970, p. 147. SAuPIN Guy, Nantes au XVII siècle..., op. cit., p. 26-28.

48. SAupIN, Guy, Ibid., p. 26-28. 
Les conseillers sont au nombre de cinq. Les quatre premiers sont classés par rang (du $1^{\mathrm{er}}$ au $4^{\mathrm{e}}$ ), quant au cinquième il porte le titre de conseiller garde-scel. Ces personnages représentent la moitié du corps en exercice. C'est par ces charges que se fait le plus souvent l'entrée dans l'administration de la communauté. La fonction de conseiller garde-scel apparaît en 1701 et comme son nom l'indique, il a en charge le cachet qui scelle les actes de la municipalité, mais ce titre est surtout honorifique.

Lors des séances du corps de la communauté, la voix de ces hommes a la même valeur. Quand une proposition est mise au vote, celle du quatrième conseiller compte autant que celle du maire ou du premier échevin. La classification des membres n'est faite que dans le but d'établir une hiérarchisation des officiers municipaux et cette hiérarchie est indispensable pour le bon déroulement des séances de travail de l'institution.

Toutefois, les échevins et les conseillers n'ont pas un rôle primordial dans le fonctionnement de la communauté. S'ils participent aux prises de décisions lors des réunions du corps de ville, s'ils prennent part épisodiquement au tribunal de police et s'ils assurent de temps à autre la police des marchés, ils ne se voient jamais confier de mission particulière. Le maire, avec l'appui du procureur-syndic et du greffier, gère et assure la grande majorité de l'activité municipale, l'action des échevins et conseillers se limitant le plus souvent à une présence aux séances et une participation aux discussions.

Des anciens membres en exercice, un miseur et un greffier complètent les composantes du corps de ville. Si l'ancien corps a un pouvoir décisionnaire, le miseur et le greffier sont deux agents techniques, en charge respectivement, du secteur financier et du secteur administratif.

L'ancien corps se constitue des anciens maires, échevins, procureurs et conseillers qui ont quitté le corps en exercice. Tous membres honoraires, ils participent au débat et prennent part aux décisions. L'appartenance à l'ancien corps est automatique pour le maire à la fin de son mandat. Pour les autres membres du corps de ville, il faut en faire la demande et ces requêtes d'entrée au corps ancien sont étudiées en séance du conseil et donnent lieu à acceptation ou à refus. Le même procédé est toujours appliqué, à l'exemple de ce qui se passe en mai 1775 .

Les faits sont évoqués lors d'une réunion du corps municipal : "Monsieur le Maire prie Messieurs de l'assemblée de délibérer sur la remontrance mise sur le registre par M. Berubé de Costentin le 17 du mois dernier $^{49}$. "Puis la demande est mise aux voix et le résultat apparaît sous la forme d'une délibération : "La Communauté a accepté la démission de Monsieur Berubé de Costentin de sa place de premier échevin en exercice et a agréé sa déclaration de passer dans l'ancien corps [...] A arrêté au surplus que la présente délibération sera adressée à son Altesse Sérénissime Monseigneur le Duc de Penthièvre pour obtenir son approbation. »

49. Arch. mun. de Brest, BB27-15, séance du 6 mai 1775. 
Selon les périodes, le nombre de membres de l'ancien corps oscille entre trois et onze personnes. Plusieurs raisons expliquent cette variation. Certains meurent, d'autres démissionnent prétextant un âge avancé ou des occupations professionnelles trop prenantes, d'autres enfin quittent Brest. Le renouvellement de l'ancien corps est également tributaire de la composition du corps en exercice. Quand un nouveau poste d'échevin ou de conseiller est attribué suite à un décès, il n'y a pas de glissement vers l'ancien corps. Il en est de même quand la reconduction des membres en exercice n'est que partielle. L'assiduité de ces hommes aux séances du conseil est très variable. La participation des anciens maires est la plus importante. Quant aux autres, ils suivent souvent de très loin l'avancement des travaux. Sur l'ensemble des membres de l'ancien corps, la moyenne de présence est de $35 \%{ }^{50}$. De plus, d'une séance à une autre, ce sont rarement les mêmes personnes qui participent aux débats proposés.

Quelques exemples montrent également que des notables brestois passent du corps en exercice à l'ancien corps puis reviennent au corps en exercice, ce qui permet de dégager les personnalités qui comptent le plus. Jean-Pierre Lunven-Kerbizodec participe au corps de ville de janvier 1751 à janvier 1771 et au cours de ces vingt ans, il transite deux fois ${ }^{51}$ par l'ancien corps. Ce n'est pas un cas isolé, car nous notons le même cheminement pour François Raby, Jean-Jacques Le Normand, Antoine Raby ou François Jourdain. Ce va-et-vient trouve son explication dans le fonctionnement interne de l'institution. Les exemples cités ci-dessus montrent trois formes de cas. Tout d'abord, les maires, à la fin de leur mandat, intègrent de droit l'ancien corps, mais quand ils sont rappelés à la tête de la mairie, ils rejoignent immédiatement le corps en exercice; c'est le cas de Le Normand et de Lunven-Kerbizodec. Pour François et Antoine Raby, la situation est un peu différente car c'est à leur demande qu'ils sont passés du corps en exercice à l'ancien corps, et quand ils ont été élus au poste de maire, ils sont donc revenus vers le corps principal de la communauté. Quant à François Jourdain, après avoir pris l'initiative de rejoindre les rangs de l'ancien corps où il demeure pendant six ans, il est de nouveau coopté en 1754 en tant que second échevin; cette situation vaut également pour JeanMarie Kerbrezan-Cabon en 1771. Le mouvement entre les deux corps laisse apparaître une certaine liberté dans la formation du groupe des officiers municipaux, aucune règle n'interdisant le passage d'une entité à une autre. Le passage dans l'ancien corps peut presque apparaître comme un repli momentané ou une position d'attente pour certains.

À travers les délibérations, il apparaît toutefois que les membres de l'ancien corps, quand ils sont présents, participent activement aux débats. Forts de leur expérience, leurs avis sont écoutés et plusieurs conseillers en exercice suivent leurs opinions, notamment celles des anciens maires qui connaissent très bien certains dossiers.

50. Calcul établi sur une période allant de janvier 1751 à mars 1790 .

51. Il intègre l'ancien corps de janvier 1757 à février 1758 et de janvier 1763 à mars 1769 . 
Corps en exercice et ancien corps forment le corps de ville décisionnaire dont tous les membres ont voix délibérative. Ce qui n'est pas le cas du greffier et du miseur qui ne sont que deux agents techniques, sans influence réelle sur les orientations municipales.

Dans la seconde moitié du XVIII ${ }^{\mathrm{e}}$ siècle, Brest ne connaît que deux miseurs : Jacques Lamothe et Julien Le Cain ${ }^{52}$. Le miseur s'occupe des deniers de la commune, il gère les finances et contrôle les octrois. Sous la coupe directe de l'intendant de Bretagne, il rend annuellement ses comptes. Gérant de la caisse de la ville, il ne peut sortir le moindre argent sans l'aval de l'autorité supérieure. Pour cette fonction, il est directement intéressé aux entrées et perçoit deux sols pour chaque livre qui rentre dans les caisses de la communauté. Comme les charges de justice, cet office s'acquiert moyennant finance. Le détenteur doit être reçu par la cour royale et par la municipalité. Quand Le Cain se présente devant la communauté de ville en 1785, l'acte de réception rappelle ses fonctions sous cette forme : " avocat à la cour contrôleur et receveur des domaines du Roi à Brest, lequel en qualité de porteur de procuration et de représentant le miseur de cette ville a remontré que suivant les édits et arrêts du conseil du mois de juillet 1694 et deux avril 1726 il est en droit de jouir des honneurs fonctions privilèges séances et autres prérogatives accordés au titulaire des offices de miseur ${ }^{53}$ ". Agent technique, le miseur n'assiste que très épisodiquement aux séances du conseil à Brest, il n'a pas la prérogative de participer aux débats - ce qui est le signe d'une ville importante -, à la différence de plusieurs villes de Bretagne où il a voix délibérative ${ }^{54}$.

De 1750 à la Révolution, Louis La Houssaye, Noël Le Ru, Claude Camus, Charles Leyrot, Pierre Gérard, Pierre Branellec et Florentin Le Bronsort occupent successivement le poste de secrétaire-greffier pour la municipalité brestoise. Le greffier est salarié de la communauté. Toujours de formation juridique, il se charge d'enregistrer les textes, gère le courrier, rédige une partie des procès-verbaux de délibérations et assiste le maire et le procureur-syndic dans le travail administratif. Il est nommé par le conseil sur proposition du maire. Mais à la moindre erreur, le poste est mis en balance. Certains en font l'amère expérience, comme en $1760^{55}$, où le corps de ville se voit contraint par l'intendant de Bretagne de nommer un nouveau greffier, car Noël Le Ru a commis des erreurs et des fautes qui n'ont pas plu au commissaire du Roi ${ }^{56}$. La palme de la longévité revient à Florentin Le

52. Lamothe exerce cette fonction de mai 1751 à novembre 1784 ; quant à Le Cain, il lui succède officiellement en décembre 1785 après avoir fait fonction pendant dix mois.

53. Arch. mun. de Brest, BB25, séance du 3 décembre 1785.

54. LE GofF, Samuel, "Finances royales et organisation politique urbaine dans les petites villes bretonnes au XVIII ${ }^{\mathrm{e}}$ siècle ", dans SAuPIN, Guy (dir.), Le Pouvoir urbain dans l'Europe atlantique du XVI $T^{\mathrm{e}}$ au XVIII . Colloque Nantes janvier 2000, Nantes, Ouest-éditions, 2002, p. 115-132.

55. Arch. mun. de Brest, BB20, séance du 12 juillet 1760.

56. Dans les archives, les erreurs commises et les reproches faits à Le Ru ne sont pas identifiables. 
Bronsort qui reste en poste d'août 1770 à décembre 1792. C'est d'ailleurs au cours de cette période, en 1775 , que le greffier cumule pour la première fois sa fonction avec celle de conseiller garde-scel ${ }^{57}$, car dans la réalité, le sceau de la ville était détenu par le greffier et non pas par un conseiller qui n'en avait que le titre. En décidant ce regroupement, le maire Jean-Jacques Le Normand a officialisé une pratique qui existait depuis le début du XVIII ${ }^{\mathrm{e}}$ siècle. Le secrétaire reste salarié du corps de ville mais il en devient aussi un des membres avec voix délibérative.

Ces deux agents de l'administration de la ville sont des éléments de la vie municipale. S'ils ne sont pas toujours membres délibérants, s'ils n'entrent pas à proprement parler dans la carrière politique, ils essayent néanmoins de servir au mieux les intérêts de la ville et de la municipalité, s'identifient à elle et ont un rôle fondamental pour son bon fonctionnement.

\section{D'un maire à l'autre, d'un conseil à l'autre}

Dans la constitution du corps en exercice, aucune règle ne définit le principe de renouvellement des membres. Hormis le maire sortant qui intègre l'ancien corps, les autres officiers municipaux peuvent demeurer dans l'institution, en restant à leur poste ou en connaissant une promotion dans la hiérarchie interne. Ce principe, appliqué durant toute la seconde moitié du XVIII ${ }^{\mathrm{e}}$ siècle, a permis à certains notables de faire carrière dans l'administration municipale, tandis que d'autres se sont limités à un passage éclair.

Taux de renouvellement des corps en exercice (1751-1789)

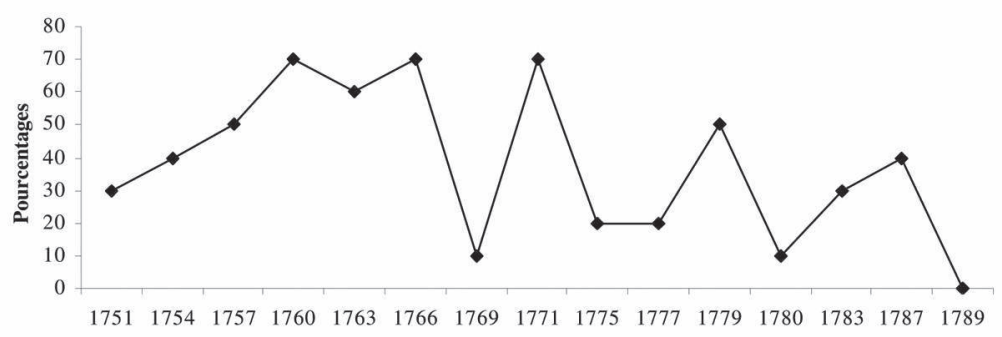

Le taux moyen de renouvellement des corps en exercice qui se succèdent de janvier 1751 à juin 1789 est de $38 \%$. Les chiffres oscillent entre $10^{58}$ et $70 \%$. En 1760,1766 et 1771 , sept nouveaux officiers municipaux

57. Arch. mun. de Brest, BB23, délibération du 6 mai 1775.

58. L'absence de renouvellement en juin 1789 n'est pas caractéristique de l'Ancien Régime mais plutôt le reflet d'un instant de crise et de tension extrême car à la suite de 
sur dix font leur entrée. Cette non-reconduction d'une grande majorité de membres peut apparaître hasardeuse du fait du manque d'expérience de la vie politique chez ces sept personnes. Mais parmi eux, tous ne sont pas des novices dans la gestion des affaires publiques car certains font leur retour après un passage dans l'ancien corps.

\section{Part des nouveaux venus dans le renouvellement des corps en exercice (1751-1789)}

\begin{tabular}{|c|c|c|c|c|c|}
\hline Année & $\begin{array}{c}\text { Part des } \\
\text { nouveaux }\end{array}$ & Année & $\begin{array}{c}\text { Part des } \\
\text { nouveaux }\end{array}$ & Année & $\begin{array}{c}\text { Part des } \\
\text { nouveaux }\end{array}$ \\
\hline 1751 & 3 sur 3 & 1766 & 6 sur 7 & 1779 & 5 sur 5 \\
\hline 1754 & 1 sur 4 & 1769 & 0 sur 1 & 1780 & 0 sur 1 \\
\hline 1757 & 5 sur 5 & 1771 & 6 sur 7 & 1783 & 2 sur 3 \\
\hline 1760 & 4 sur 7 & 1775 & 1 sur 2 & 1787 & 4 sur 4 \\
\hline 1763 & 6 sur 6 & 1777 & 0 sur 2 & 1789 & $\begin{array}{c}\text { Pas de } \\
\text { renouvelle- } \\
\text { ment }\end{array}$ \\
\hline
\end{tabular}

La variation des renouvellements a plusieurs explications. Les départs volontaires représentent les motifs les plus importants. Après plusieurs années passées au sein du corps en exercice, des officiers municipaux aspirent à se retirer de la vie publique ou à passer dans l'ancien corps. Las de la gestion de la ville, ils se recentrent sur leurs affaires. Certains n'ont plus assez de temps à donner, devant gérer de front leur carrière et faire face à des besoins familiaux. D'autres se retirent pour bénéficier d'un repos après une activité politique et professionnelle plus ou moins longue. L'âge est également un des critères les plus avancés pour pouvoir démissionner.

Parfois le renouvellement est important car la situation l'exige. En 1760, l'apparition de sept nouveaux membres par rapport au corps précédent s'explique par le décès du procureur-syndic et du $1^{\mathrm{er}}$ conseiller, la démission des $2^{\mathrm{e}}$ et $3^{\mathrm{e}}$ conseillers, le départ momentané de Brest pour affaires du premier échevin et du conseiller garde-scel et le passage dans l'ancien corps du maire. En 1766, plusieurs membres du corps en exercice ont préféré ne pas prolonger leur expérience car durant le mandat du maire Charles Féburier, les relations ont été très tendues au sein du conseil de la communauté; ces personnes, se sentant éprouvées, préfèrent ne pas renouveler l'expérience.

Au sein du corps en exercice, il est également intéressant de connaître la répartition des postes entre le côté de Brest et celui de Recouvrance. La règle de l'alternance pour le poste de maire est définie depuis 1687 mais

l'élection du maire et face aux événements en cours, les officiers municipaux avaient décidé de ne pas coopter de nouveaux membres. 
en ce qui concerne les autres fonctions, rien n'est établi. Durant toute la seconde moitié du XVIII ${ }^{\mathrm{e}}$ siècle, la répartition des dix membres du corps est de 6-7 pour Brest contre 3-4 pour Recouvrance. Ce ratio correspond environ à la distribution de la population sur l'ensemble du territoire mais surtout à la présence des hommes capables d'intégrer le corps de ville puisque la grande majorité des notables disposant d'une assise financière et publique se trouve sur la rive gauche de la Penfeld. Les négociants, marchands, hommes de loi, professions médicales y sont beaucoup plus nombreux que sur la rive droite. C'est le côté des affaires et des centres décisionnaires, à la différence du quartier de Recouvrance qui est considéré comme étant le lieu de vie de la masse des ouvriers et petits métiers.

Durant les quarante dernières années de l'Ancien Régime, les postes majeurs du corps en exercice sont détenus majoritairement par des résidents du côté de Brest : 66,7 \% des maires, 62,5\% des échevins et 83,4 \% des procureurs du roi. Il arrive même que ces quatre places soient monopolisées par des notables de la rive gauche comme de juin 1766 à mars 1769. Cette situation met en avant la difficulté à trouver des personnes de Recouvrance pouvant occuper les hautes fonctions municipales. Les hommes disposant de la fortune et des capacités nécessaires ne sont pas très nombreux et doivent se maintenir en place durant une assez longue période afin que la rive droite soit représentée. Ce qui explique que sur l'ensemble des changements de personnel intervenus durant la seconde partie du XVIII ${ }^{\mathrm{e}}$ siècle, le taux de renouvellement pour les membres de Recouvrance n'est que de 38,6 \%, contre $61,4 \%$ au côté de Brest.

Tous les hommes ayant fréquenté le corps en exercice durant au moins deux périodes de renouvellement ont connu une promotion dans la hiérarchie municipale ${ }^{59}$. Les maires ont occupé au moins deux autres postes avant d'être élus. Par contre, aucune règle ne se dégage pour se retrouver premier magistrat. La fonction d'échevin n'est pas un passage obligé, la moitié des maires n'ont jamais connu l'échevinage. Trois étaient au préalable procureur, et trois sont passés directement de conseiller à maire. Il n'y a donc pas de cursus obligatoire pour atteindre le poste de premier magistrat, les édiles faisant carrière au sein de l'institution sans pour autant franchir des étapes prédéfinies. La qualité de gestionnaire n'est ni un argument électif, ni de choix. Les contemporains veulent un homme disponible et, de préférence, possédant une fortune confortable. Avant de devenir maire, ces hommes ont passé entre 6 et 14 ans au sein du conseil de la communauté.

Sur les seize échevins, deux seulement ne sont pas passés par le rang de conseiller, accédant à cet échelon après avoir été procureur-syndic. Tous les autres ont été une ou deux fois conseillers. Aucun dispositif n'oblige donc l'échevin à avoir été auparavant conseiller, comme c'est le cas dans

59. Cette affirmation ne s'applique pas à Florentin Le Bronsort qui fut conseiller gardescel durant quinze années. Mais il ne pouvait pas profiter du système étant également secrétaire-greffier de la communauté. 
certaines villes du royaume telles que Marseille ${ }^{60}$. Les échevins restent en poste en moyenne cinq ans, ils accèdent aux deux niveaux du système scabinal, le second devient le premier lors du renouvellement qui suit l'élection du maire. Cette règle est appliquée sans discontinuer, sauf en 1771 et 1787. En juillet 1771, le second échevin Antoine Blad demande à être nommé premier échevin, mais lors du vote interne, il n'est pas élu à ce poste ${ }^{61}$, il doit même intégrer l'ancien corps, n'étant pas conservé dans le corps en exercice. Il aspirait au rang de premier échevin en remerciement de toutes les années passées au service de la communauté, mais malheureusement pour lui, son ambition a été mise à mal par le système électoral qui ne l'a pas porté à cette fonction tant désirée ${ }^{62}$. En 1787 , le cas opposé se présente : Charles-Louis Gillart demande à ne pas être promu premier échevin car il occupe le poste de subdélégué de l'intendant et selon ses propos ${ }^{63}$, cette fonction est devenue incompatible avec une place d'administrateur municipal $^{64}$.

De janvier 1751 à la Révolution, douze procureurs-syndics se succèdent, la durée moyenne de la fonction est de trois ans. C'est une tâche qui demande du travail et une liberté de temps puisqu'il faut avoir l'opportunité de consulter les documents et de faire des recherches dans les archives. Parmi ces douze hommes, quatre ont été substituts auparavant, les huit autres accédant à cette fonction après un poste de conseiller, ce n'est donc pas une obligation d'être substitut pour devenir procureur du roi. Une fois de plus, il n'y a pas de règle définie pour atteindre une place.

Quarante-trois hommes occupent un des cinq postes de conseillers; $32,5 \%$ connaissent une promotion interne dans les rangs des conseillers : certains passent de conseiller garde-scel à $2^{\mathrm{e}}$ conseiller ou grimpent d'une ou deux places parmi les conseillers ordinaires, $62,8 \%$ des conseillers ont exercé après un ou deux mandats une fonction plus importante au sein du corps en exercice et les $37,2 \%$ restants sont demeurés conseiller tout au long de leur carrière ou ont quitté le corps de ville après une première expérience. Pour la hiérarchie au sein du corps en exercice, aucune règle ne définit des étapes obligées, on accède à un certain rang en fonction de ses capacités mais aussi par rapport aux besoins présents. Il n'y a pas obligation d'être substitut pour devenir procureur-syndic et un maire n'a pas été nécessairement échevin.

Plusieurs de ces notables ont passé une grande partie de leur existence à fréquenter le corps de ville, le record de longévité, 31,5 ans, est détenu

60. EmMANUELLI, François-Xavier, "La municipalité de Marseille et l'État royal à la fin du XVIII ${ }^{\mathrm{e}}$ siècle ", Marseille, face au(x) pouvoir(s). Actes du colloque Marseille 4 et 5 février 2000, Marseille, Archives départementales des Bouches-du-Rhône, 2002, p. 63-81.

61. Arch. mun. de Brest, BB22, séance du 13 juillet 1771.

62. Dans les archives, aucun document ne permet d'expliquer la non-promotion de Blad et son versement dans l'ancien corps.

63. Arch. mun. de Brest, BB25, séance du 13 septembre 1787.

64. Il est subdélégué depuis 1783 et a fait son entrée au corps de ville en 1771 . 
conjointement par Charles Féburier et Antoine Raby neveu. Certains ont passé plus de temps dans l'ancien corps que dans le corps en exercice, mais la période au sein de l'ancien corps ne correspond pas à un grand cycle d'activités. Ces hommes sont souvent absents et n'assistent qu'à de très rares séances, notamment celles où il faut choisir les candidats pour le poste de maire ou voter pour attribuer les fonctions communautaires.

François-Marie Guesnet est l'officier municipal qui a assuré la plus longue période (19 ans) sans discontinuer au sein du corps en exercice. Il est suivi par deux maires : Pierre Betbédat et François Le Guen, qui, après 17 ans de service, se sont retirés dans l'ancien corps. Mais sur l'ensemble du personnel municipal de la seconde moitié du xVIII ${ }^{\mathrm{e}}$ siècle, ces exemples sont des exceptions car la moyenne de présence correspond à huit années, $24,1 \%$ des officiers ne demeurent en poste que lors d'un mandat ${ }^{65}$ et disparaissent par la suite de la vie municipale, ces hommes se rapprochant des " météores politiques " de Guy Saupin, 33,33 \% restent le temps de deux mandats et d'autres participent plus longuement aux activités de la communauté : $22,2 \%$ ont exercé trois mandats et $20,4 \%$ en ont fait au moins quatre. Une majorité de ces hommes le font par choix, tandis que d'autres, comme beaucoup de notables de Recouvrance, persistent afin de maintenir une certaine représentation, mais aussi car à cette époque, la notion d'honneur est beaucoup plus marquée que l'appât du gain ${ }^{66}$.

À l'image de la société urbaine du royaume, la cité brestoise est donc administrée par un corps municipal composé d'un corps en exercice et d'un ancien corps. Cette organisation bi-corporelle se retrouve dans plusieurs villes du royaume, comme Nantes avec son Grand Bureau constitué d'un bureau servant et d'un ancien bureau ${ }^{67}$ ou Lorient où tous les officiers sortis de charge restent présents au sein de la municipalité ${ }^{68}$. L'amalgame entre nouveaux et anciens officiers résulte du souhait d'assister à une continuité dans la gestion des affaires. Brest s'établit comme une ville d'échevinage comme celles de la moitié nord de la France (Saint-Brieuc, Rennes, Caen, Rouen, Reims, Paris, Angers etc.) à la différence de celles des consulats (sud), jurats (Bordeaux, Libourne, Blaye etc.), capitouls (Toulouse) ${ }^{69}$.

Le maire est élu par une assemblée générale regroupant des représentants des corporations de métiers, ce qui n'est pas une tradition de l'Ouest, et les forces d'encadrement qui contrôlent la population sur un plan politique, économique, juridique et spirituel. La composition de cette

65. On entend par mandat une période de trois ans, équivalent à la durée d'exercice initiale de la fonction de maire.

66. Coste, Laurent, Les Lys et le chaperon. Les oligarchies municipales en France de la Renaissance à la Révolution, Bordeaux, PU Bordeaux, 2007, p. 25.

67. SAUPIN, Guy, Nantes au XVIז siècle..., op. cit., p. 19.

68. Kerloc'H, Marianne, Les élites municipales de Lorient (1738-1789), TER (dir. Gérard Le Bouëdec), Lorient, UBS, dactyl., 2001, p. 17.

69. Coste, Laurent, Les Lys et le chaperon..., op. cit., p. 135. 
assemblée, tout comme à Lorient ${ }^{70}$, n'est que le pâle reflet de la structure socio-démographique de la population.

Aucune uniformisation de désignation du maire n'existe en France sous l'Ancien Régime. À Brest, le choix du maire est entériné par le gouverneur, après une assemblée générale fermée qui décide, à partir d'une liste de trois postulants, qui va occuper la place. Certaines villes comme La Rochelle, Tours, Angers, Caen, Alençon, Amiens ${ }^{71}$ élisent tout d'abord des candidats puis une autorité supérieure, telle que le roi ou le gouverneur de la province, choisit le maire. Dans ce sens, les représentants brestois ont la particularité de pouvoir désigner directement celui qui exerce la fonction; ce même cas de figure existe aussi à Poitiers, à Abbeville ou à Dax ${ }^{72}$.

L'ensemble du corps de ville ne comprend que dix officiers en exercice, plus une poignée d'anciens membres. Ce chiffre est très loin de la réalité de cités comme Bordeaux ou Nantes. L'importance de l'administration municipale ne tient pas compte de la démographie de la ville. À la veille de la Révolution, Morlaix ${ }^{73}$ dispose d'un nombre d'officiers municipaux identique à celui de Brest alors que la ville est de moindre importance. De même, Lorient compte deux fois plus d'officiers municipaux que Brest ${ }^{74}$. $\mathrm{Au} \mathrm{XVIII}$ siècle, le rapport quantitatif entre population et administration locale n'est pas établi.

Cela montre aussi qu'une minorité de personnes détient le pouvoir au détriment des couches moyennes et populaires. La municipalité brestoise, comme celle des autres villes du royaume, ne reflète pas la composition des sociétés urbaines. Toutefois, l'importance de ce pouvoir doit être relativisée du fait de la présence constante des autorités militaires qui sont les réels détenteurs du pouvoir dans la cité et si l'intendant de la province éprouve quelques difficultés à asseoir son autorité, il n'en reste pas moins un élément majeur dans la tutelle administrativo-étatique.

70. Kerloc'H, Marianne, Les élites municipales de Lorient (1738-1789), op. cit., p. 44

71. Delafosse, Marcel (dir.), Histoire de La Rochelle, Toulouse, Privat, 1985, p. 160. BAUMIER, Béatrice, Tours entre Lumières et Révolution. Pouvoir municipal et métamorphoses d'une ville (1764-1792), Rennes, PUR, 2007, p. 42. LEBRUn, François (dir.), Histoire d'Angers, Toulouse, Privat, 1975, p. 107. PATRY, Robert, Une ville de province. Caen pendant la Révolution de 1789, Condé-sur-Noireau, Corlet, 1983, p. 101. RugGiU, François-Joseph, Les Élites et les villes moyennes en France et en Angleterre (XVII ${ }^{e}$-XVIII $I^{e}$ siècles), Paris, L'Harmattan, 1997, p. 33. HuBSCHER, Ronald (dir.), Histoire d'Amiens, Toulouse, Privat, 1986, p. 176.

72. GUIGNET, Philippe, Les Sociétés urbaines dans la France moderne, Paris, Ellipses, 2006, p. 56. RugGiu, François-Joseph, ibid., p. 30. PONTET, Josette, " Officiers et corps de ville à Dax au XVIII ${ }^{\mathrm{e}}$ siècle ", CASSAN, Michel (dir.), Les officiers "moyens " à l'époque moderne : pouvoir, culture, identité. Actes du colloque Limoges avril 1997, Limoges, PU Limoges, 1998, p. $97-120$.

73. BoRDES, Maurice, L'Administration provinciale et municipale en France au XVIII e siècle, Paris, La Sorbonne, 1972, p. 217.

74. NiÈRES, Claude (dir.), Histoire de Lorient, Toulouse, Privat, 1988, p. 104. 
En appliquant le principe de la cooptation, processus le plus répandu en France ${ }^{75}$, les possibilités d'intégrer le conseil de la communauté sont restreintes. Sans reconnaissance professionnelle, sans réussite sociale, voire sans appui, pénétrer dans le corps de ville représente un objectif irréalisable. De plus, la pérennité de la fonction - on est membre du conseil à vie - limite quelque peu les possibilités de renouvellement du corps de la communauté, même si celui-ci est bien présent avec un taux relativement élevé de $35 \%$. Les postes n'étant pas limités dans le temps, certains membres ne quittent le conseil qu'après une ou plusieurs décennies. Par contre, à Brest, il n'y a pas de conditions d'âge, de statut ou de résidence pour intégrer le corps de la communauté à la différence de villes comme Aix-en-Provence ou Reims ${ }^{76}$. Le lieu de naissance et la date d'installation dans la cité ne rentrent pas en ligne de compte pour intégrer l'administration locale. Il suffit avant tout de bénéficier d'un statut social assez élevé et de la reconnaissance de ses pairs.

Mais la Révolution va changer cette donne dans la hiérarchie des pouvoirs. En 1789, les cahiers de doléances brestois appelleront à une modification des administrations urbaines et les élections du printemps 1790 apporteront à la ville une nouvelle forme de municipalité, avec une très grande majorité d'hommes nouveaux.

75. Coste Laurent, Les Lys et le chaperon..., op. cit., p. 135.

76. DEROBERT-RATEL Christiane, « La classe politique aixoise de la fin de l'Ancien Régime à l'an VIII ", Annales du Midi. Revue de la France méridionale, n ${ }^{\circ} 151,1981$, p. 51-69. Hourbuin Xavier, Les Finances de Reims à la fin de l'Ancien Régime. 1765-1789, Paris, CHEFF, 2008, p. 21. 


\section{RÉSUMÉ}

Dans la seconde moitié du $\mathrm{XVIII}^{\mathrm{e}}$ siècle, la ville de Brest est administrée par un corps de ville qui comprend deux entités : le corps en exercice (maire, échevins, procureur syndic, conseillers) et l'ancien corps (membres ayant quitté le corps en exercice).

L'histoire de cette institution est assez récente car la première véritable organisation municipale n'apparaît qu'à la fin du Xvi ${ }^{\mathrm{e}}$ siècle et connaît jusqu'en 1750 quelques modifications et ajustements.

L'élément essentiel de cette administration est le maire, sur qui repose la majeure partie du travail. De la nature et de la motivation de cet individu dépend le dynamisme influé à la cité du Ponant. Il est aidé ponctuellement dans sa tâche par neuf autres officiers municipaux, qui sont cooptés à la différence du maire qui lui, est élu par une assemblée générale.

Ces édiles forment un groupe d'élites municipales. Cependant, si les carrières et les promotions semblent importantes au sein du corps de ville, ces personnages demeurent en retrait des nombreuses autorités nobiliaires présentes à Brest et ne peuvent rivaliser dans leurs actions avec le pouvoir des militaires qui constituent la véritable élite sociale.

\section{ABSTRACT}

In the second half of the 18th century, the city of Brest was administered by a municipal corporation that included two entities: the active corps (mayor, aldermen, elected prosecutor, councillors) and the old corps (past members).

The history of this institution was rather recent as the first real municipal organisation only appeared at the end of the 16th century and was modified and adjusted up until 1750.

The essential element of this administration was the mayor who was responsible for most of the work. The dynamism of the western city depended on the character and motivation of this individual. He was occasionally helped in his tasks by nine other municipal officers who were co-opted, unlike the mayor who was elected by a general assembly.

These officials formed a municipal elite. However, if careers and promotions seemed important within the municipal corporation, these individuals remained on the side-lines when compared to the numerous noble authorities present in Brest and could not compete in their actions with the power of the military that constituted the real social elite. 